\title{
54. LABORATORY SIMULATION OF ICY COMETARY NUCLEI
}

\author{
E. A. KAJMAKOV and V. I. SHARKOV \\ A. F. Ioffe Institute of Physics and Technology, Leningrad, U.S.S.R.
}

\begin{abstract}
The problems of laboratory simulation of cometary phenomena are discussed. Results are given of experimental investigations of the thermodynamic parameters of $\mathrm{H}_{2} \mathrm{O}$ ice and of frozen mixtures of electrolytes during sublimation under conditions of high vacuum and low temperature. At a distance of $1 \mathrm{AU}$ from the Sun the equilibrium temperature of $\mathrm{H}_{2} \mathrm{O}$ ice is shown to be about $-75^{\circ} \mathrm{C}$. Conditions are determined for the formation and disintegration of dust matrices during the sublimation of ices containing small dust particles. Dust particles are shown to leave the surface of the ice in the form of conglomerates of primary particles. The outflow velocities of the particles are found to be as high as several metres per second. The results obtained are discussed in relation to cometary phenomena.
\end{abstract}

The great strides made by man in gaining mastery over cosmic space have been responsible for the vigorous development of research in astrophysics in many directions. Because of their rapid variations, comets are of particular interest to astrophysicists. Since comets are sensitive to electrical and magnetic fields and to the solar wind they can be visualized as cosmic space probes. Observations of changes in their shapes and sizes, as well as in their spectra, permit us to study conditions prevailing in regions of space as yet inaccessible to man-made space probes (Beller, 1963).

According to the most widely accepted hypothesis, cometary nuclei consist of mixtures of various ices and particles of high melting point. Solar radiation causes sublimation of the ices, accompanied by ejection of the particles. There are many additional factors that greatly complicate the picture.

Most of the molecules, atoms and ions present in cometary atmospheres are formed as a result of ionization and dissociation of primary, or 'parent' molecules. Among these parent-molecules are $\mathrm{H}_{2} \mathrm{O}, \mathrm{CO}_{2}, \mathrm{NH}_{3}, \mathrm{CH}_{4},(\mathrm{CN})_{2}$ and several other organic and inorganic molecules of greater or less complexity (Donn, 1964). The best defined features in cometary spectra are bands corresponding to $\mathrm{CN}, \mathrm{C}_{2}, \mathrm{OH}$ and other molecules.

Many cometary phenomena await clarification. There are still a great many unsolved problems relating to the compactness and chemical composition of the nucleus, formation of the dust component in the coma, outbursts in brightness, etc. Individual hypotheses fail to explain all the observed facts.

Experimental research on comets is nowadays being conducted in many directions, using up-to-date techniques and modern instrumentation. We make particular reference to the use of telescopes in conjunction with sophisticated spectrographs and image converters, permitting radical reductions in exposure times; this method has already yielded important results (Rylov, 1967). Another new technique is the use of satellites and spacecraft for photography and spectroscopy of comets in the ultraviolet and infrared. Such studies ensure freedom from the influence of the Earth's atmosphere, enabling new data to be obtained and earlier results to be deciphered (Boggess, 1965). 
There is also the possibility of launching automatic probes towards comets. Plans currently under discussion in various countries envisage a flight around a comet, photography of the nucleus, sampling the atmosphere, and determination of the ionic composition (Haser and Lüst, 1966; Roberts, 1963).

Yet another direction of study is concerned with the simulation of cometary phenomena; this can be realized in laboratory conditions as well as out in space. Here we may include experiments in producing artificial clouds at high altitudes, observing the motions and changes in configuration of these clouds, and obtaining spectrograms. Experiments have been conducted with $\mathrm{Na}$ (Shklovskij and Kurt, 1959), $\mathrm{NH}_{3}$ (Rosen and Bredohl, 1965), Ba and $\mathrm{Sr}$ (Föppl, 1965) and $\mathrm{H}_{2} \mathrm{O}$ clouds, and many interesting results have been obtained. It will be noted, however, that such experiments are only indirectly associated with cometary problems, for they are conducted mainly for the study of the upper atmosphere. The molecules of most direct interest to cometary investigators have not yet been considered.

The possibility of creating an artificial cometary nucleus has more than once come under discussion, although it is not at all clear how or of what material it should be made. Some astronomers have suggested launching into space an icy rock (Swings, 1962), while others have proposed a ton of $\mathrm{CO}_{2}$ or $\mathrm{NH}_{3}$ (Shklovskij and Kurt, 1959). Launching such a large object into space would be a fairly involved and costly experiment, and we do not see that it would provide a great wealth of information. Considerable preparatory work would be necessary for the selection of suitable materials for both the gas and dust components, and at the same time, a great many technical problems would have to be solved.

Laboratory simulation of cometary phenomena has been conducted on a limited scale. The difficulty here is to provide conditions close to those of outer space. The solar wind and solar electromagnetic radiation interact with the gaseous component of a cometary atmosphere, causing ionization and photodissociation of the parentmolecules. Laboratory studies by Potter and del Duca (1964) have narrowed down the range of possible parent substances for the observed cometary radicals. Their experiments indicate that at a distance of $1 \mathrm{AU}$ from the Sun simple compounds yielding $\mathrm{C}$, $\mathrm{CH}$ and $\mathrm{C}_{2}$ usually have life spans greater than $10 \mathrm{~h}$, whereas in order to explain the observations the life spans should be an order of magnitude smaller. Thus it seems that pure ices (or mixtures of them) cannot be parent substances for the $\mathrm{C}-, \mathrm{C}_{2^{-}}$, and $\mathrm{CH}$ groups, and a search is indicated for other, less stable substances.

A very interesting experiment that bears on the problem of the origin of cosmic ices has been performed by Berger. This consisted of subjecting a frozen mixture of $\mathrm{H}_{2} \mathrm{O}, \mathrm{CH}_{4}$, and $\mathrm{CH}_{2}$ at a temperature of $-230{ }^{\circ} \mathrm{C}$ to proton radiation from a cyclotron. Chemical analysis of the resultant products revealed traces of $\mathrm{CO}\left(\mathrm{NH}_{2}\right)_{2}$ (urea), $\mathrm{CH}_{3} \mathrm{COOH}$ (acetic acid) and $\mathrm{CH}_{3} \mathrm{COCH}_{3}$ (acetone). Experiments such as this show that it is not at all impossible that fairly complex chemical compounds may be formed during the evolution of cometary nuclei.

Another example of laboratory simulation of cometary phenomena may be seen in the work of Danielsson and Kasai (1968). Their purpose was to study the ionization of molecules and the acceleration mechanisms in cometary atmospheres, as represented 
in the interaction of $\mathrm{CO}_{2}$ ice and hydrogen plasma. As a result, they produced a 'comet tail' consisting mainly of heavy $\mathrm{CO}_{2}$ ions and found that these are accelerated by plasma flow to speeds as high as $10^{5} \mathrm{~cm} \mathrm{~s}^{-1}$, which amounts to $10 \%$ of the plasma flow velocity. This type of experiment is of considerable scientific interest.

An extensive and complex programme of studies on cometary phenomena has been carried out for a number of years at the A. F. Ioffe Institute. The studies concerned the behaviour of various types of ice (including dustladen specimens) when they are subjected to the action of conductive and radiant heat and ultraviolet radiation under conditions of high vacuum and low temperature.

The initial experiments were on pure $\mathrm{H}_{2} \mathrm{O}$ ice, of which many properties had been adequately investigated (Dorsey, 1940). An ice sample prepared from distilled water in a special cell was placed in a vacuum chamber (at $10^{-5}$ to $10^{-6} \mathrm{~T}$ ) whose walls were cooled with liquid nitrogen. Embedded in the ice sample was a miniature electric heater for supplying energy to the ice and several copper-constantan thermocouples for measuring the temperature at various points. The cell was suspended on a spring balance, which served to determine changes in the weight of the ice sample due to sublimation in the vacuum. From these experiments the following relationships and parameters were successfully determined:

(1) The relationship between the 'equilibrium' temperature $T$ of the ice and the specific power input $W_{s p}$ supplied per unit area of the ice surface (see Figure 1). The equilibrium temperature is the temperature at which the energy input is consumed solely in molecular sublimation. The practical procedure for determining this is described by Kajmakov et al. (1972). If the water-ice albedo is taken to be 0.7 , the curve may be used to determine the temperature of an $\mathrm{H}_{2} \mathrm{O}$ cometary nucleus at various

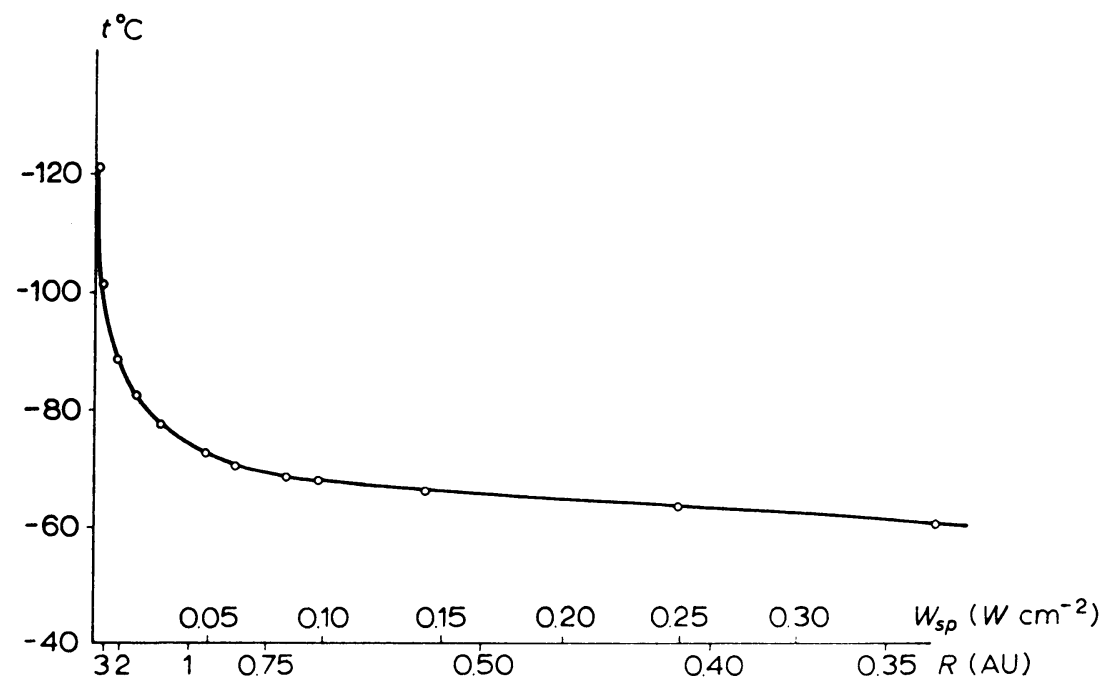

Fig. 1. Temperature of $\mathrm{H}_{2} \mathrm{O}$ ice $T$ ( C) versus specific power input $W_{s p}\left(\mathrm{~W} \mathrm{~cm}^{-2}\right)$. Corresponding heliocentric distances $r(\mathrm{AU})$ are shown for an assumed albedo 0.7 . 
distances $r$ from the Sun, provided that the comet's atmosphere does not have a shielding effect upon the nucleus. At $r=1$ AU the nuclear temperature would be $-75^{\circ} \mathrm{C}$.

(2) The ice consumption $J$ at various temperatures. The solid curve in Figure 2 shows the experimental values, while the broken curve is computed on the basis of the kinetic theory of gases.

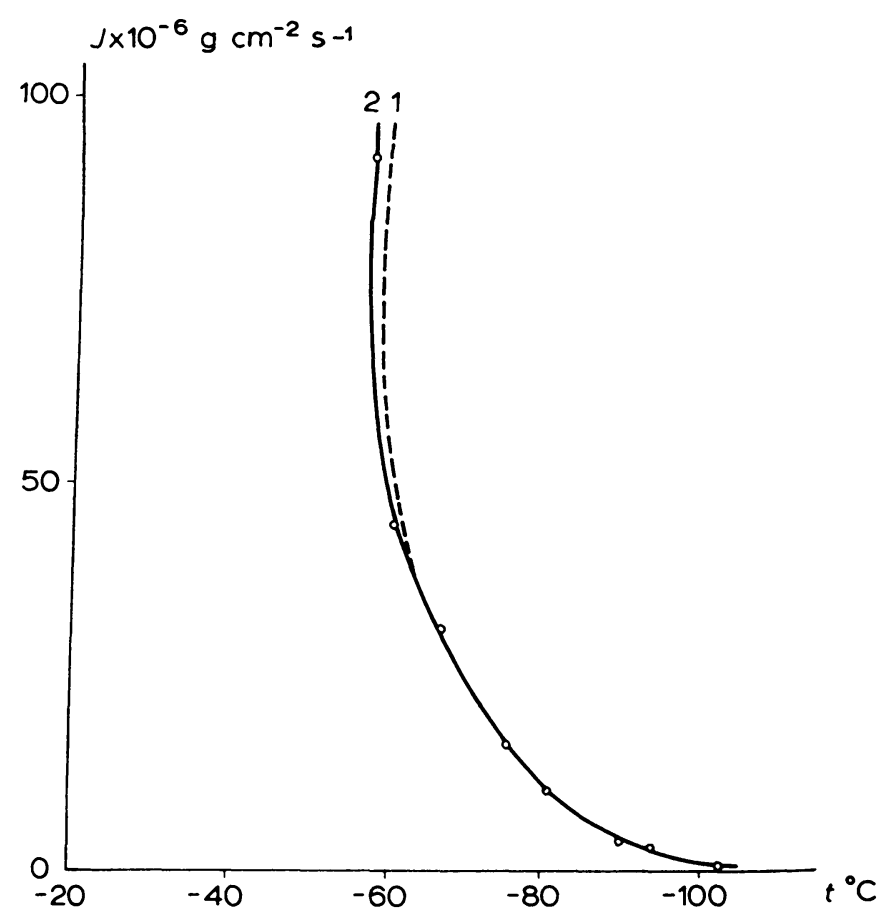

Fig. 2. Consumption rate of $\mathrm{H}_{2} \mathrm{O}$ ice $J\left(\mathrm{~g} \mathrm{~cm}^{-2} \mathrm{~s}^{-1}\right)$ versus temperature $T\left({ }^{\circ} \mathrm{C}\right)$. The broken curve (labelled 1 ) is theoretical, the solid curve (labelled 2) experimental.

(3) The heat of sublimation, i.e., the energy required for breaking the molecular bonds at the ice surface and the kinetic energy of the molecules. Within the temperature range -60 to $-90^{\circ} \mathrm{C}$ the sublimation energy was $(670 \pm 20) \mathrm{cal} \mathrm{g}^{-1}$.

We have studied the sublimation of $\mathrm{H}_{2} \mathrm{O}$ ice in a vacuum under the action of light beams. The light source used was a very high pressure mercury-quartz lamp whose energy distribution over the wavelength range 2300 to $10000 \AA$ is similar to that of sunlight. A light beam of known power was applied to the surface of the ice sample, and the ice consumption over the temperature range -55 to $-60^{\circ} \mathrm{C}$ was determined. For calculations involving the light power absorbed the ice albedo was again taken to be 0.7 , and within the limits of error the experimental results based on light absorption coincided with those based on the application of heat energy to the ice. 
In addition to experiments on pure water ice, we have conducted a series of experiments on frozen aqueous solutions of electrolytes. Studying the behaviour of such systems under conditions of high vacuum and low temperature is of interest both to the physics of solids and to the physics of comets. For instance, Delsemme and Swings (1952) are of the opinion that parent-molecules must be present in cometary nuclei, not as pure ices but as hydrates of the general form $B \cdot n \mathrm{H}_{2} \mathrm{O}$, where $B$ is the appropriate parent-molecule and $n \mathrm{H}_{2} \mathrm{O}$ is the number of water molecules with which it is combined.

By their outward behaviour in vacuo frozen solutions can be divided into two categories:

(1) those which form a porous salt matrix during sublimation, such as $\mathrm{KCl}$ or $\mathrm{CO}\left(\mathrm{NH}_{2}\right)_{2}$ solutions, and

(2) those which do not, such as $\mathrm{NH}_{4} \mathrm{OH}$ and $\left(\mathrm{NH}_{4}\right)_{2} \mathrm{CO}_{3}$ solutions.

The first category is more common. Solutions of the $\mathrm{LiCl}$ type, which contain hydrated ions, are in a special category.

Initial results have been obtained on ice consumption and temperature as a function of energy input for solutions such as $\mathrm{NH}_{4} \mathrm{OH},\left(\mathrm{NH}_{4}\right)_{2} \mathrm{CO}_{3}, \mathrm{LiCl}$ and $\mathrm{KCl}$. Experiments on frozen solutions allow us to avoid many of the difficulties associated with ices of a different chemical nature, such as $\mathrm{CO}_{2}, \mathrm{C}_{2} \mathrm{H}_{2}, \mathrm{CH}_{4}$, etc. There is an extremely wide choice of substances, and it is possible to select combinations that would produce spectra similar to those observed for real comets affected by solar radiation.

It is known that sunlight is scattered by dust particles in cometary atmospheres and tails. The mechanism of dust-particle formation and acceleration, as well as other problems relating to the behaviour of the dust component of cometary atmospheres, are of particular interest, and we have therefore studied the behaviour in vacuo of ices containing dust components of various types (Kajmakov and Sharkov, 1969).

As before, these experiments were conducted in a cooled vacuum chamber. The ice sample containing dust contaminants was prepared as follows. A quantity of dust and a predetermined volume of distilled water were placed in a special container. After being thoroughly mixed and cooled down to +1 or $+2{ }^{\circ} \mathrm{C}$, the mixture was quickly transferred to a nickel cell to be further cooled by liquid nitrogen. The cell and ice thus formed were suspended in the vacuum chamber over a glass plate. The measurements commenced when the pressure in the chamber dropped to $10^{-5}$ to $10^{-6} \mathrm{~T}$. Either heat or light energy was applied to the ice, which assumed a certain steady-state temperature, related to the energy input. At this temperature sublimation of the ice occurred, accompanied either by the outflow of dust particles and conglomerates thereof, or by the formation of a dust matrix. Dust particles and conglomerates left traces on the glass plate, and this enabled us to determine the velocities and angular distribution of the outflowing particles and the difference in size between the initial particles and those which formed the traces. The angular distribution and velocities of the outflowing particles were determined by photometry of the traces. It was established that dust particles leave the ice surface almost perpendicularly (deviating from the normal by less than $15^{\circ}$ ), and that the outflow angles are only slightly dependent on the nature, concentration and size. As the temperature of the ice increases, so does the number of 
particles with outflow angles substantially different from the normal. Figure 3 shows velocity versus ice temperature for various particles. The points labelled $M$ on each curve correspond to the formation of dust matrices, conditions for the formation of which are as follows:

(1) The lower the density of the dust particles, the smaller their size and the higher the concentration and the probability of forming a matrix.

(2) The higher the temperature of the ice, the lower the probability of matrix formation, because as the temperature increases, so does the total impulse imparted to the dust particles by the sublimation products.

(3) The higher the albedo of the dust-laden ice, the lower the probability of dustmatrix formation. This conclusion was drawn from experiments made to ascertain the influence of a light beam (wavelength 3000 to $7000 \AA$ ) upon the surface of dustladen ice.

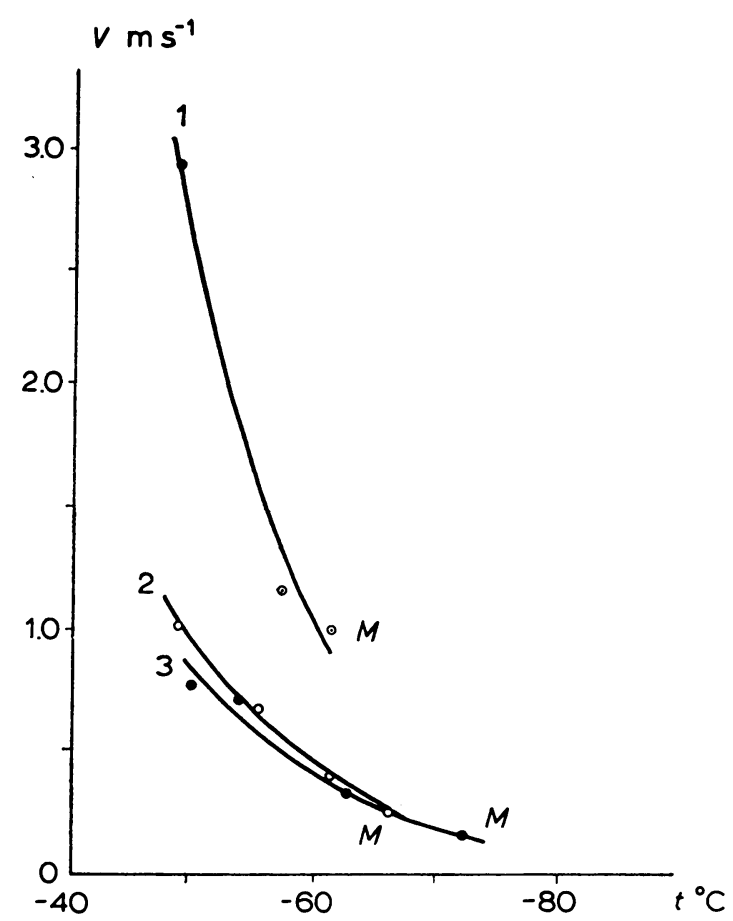

Fig. 3. Dust-particle velocity $V\left(\mathrm{~m} \mathrm{~s}^{-1}\right)$ versus surface temperature of $\mathrm{H}_{2} \mathrm{O}$ ice $T\left({ }^{\circ} \mathrm{C}\right)$ for (1) $\mathrm{Al}_{2} \mathrm{O}_{3}$, particle size $2 \mu$, volume concentration $0.35 \%$; (2) $\mathrm{Al}_{2} \mathrm{O}_{3}, 2 \mu, 1.05 \%$; (3) $\mathrm{SiO}_{2}, 20 \mu, 5 \%$; $M$ denotes the formation of a dust matrix.

If the traces left by the dust particles are examined under a microscope, the following pattern will be observed. At small distances from the icy surface the great majority of the initial particles produce conglomerates (Figure 4). Farther from the icy surface fewer form conglomerates and more separate particles are visible. The number of 
particles constituting conglomerates increases with increasing dust particle concentration and decreasing particle size and ice temperature. Particles 2 microns across and having a concentration by volume of $0.35 \%$ will form conglomerates with an average length of 5 to $7 \mathrm{~mm}$ and width 2 to $3 \mathrm{~mm}$. The data obtained permit us to make the

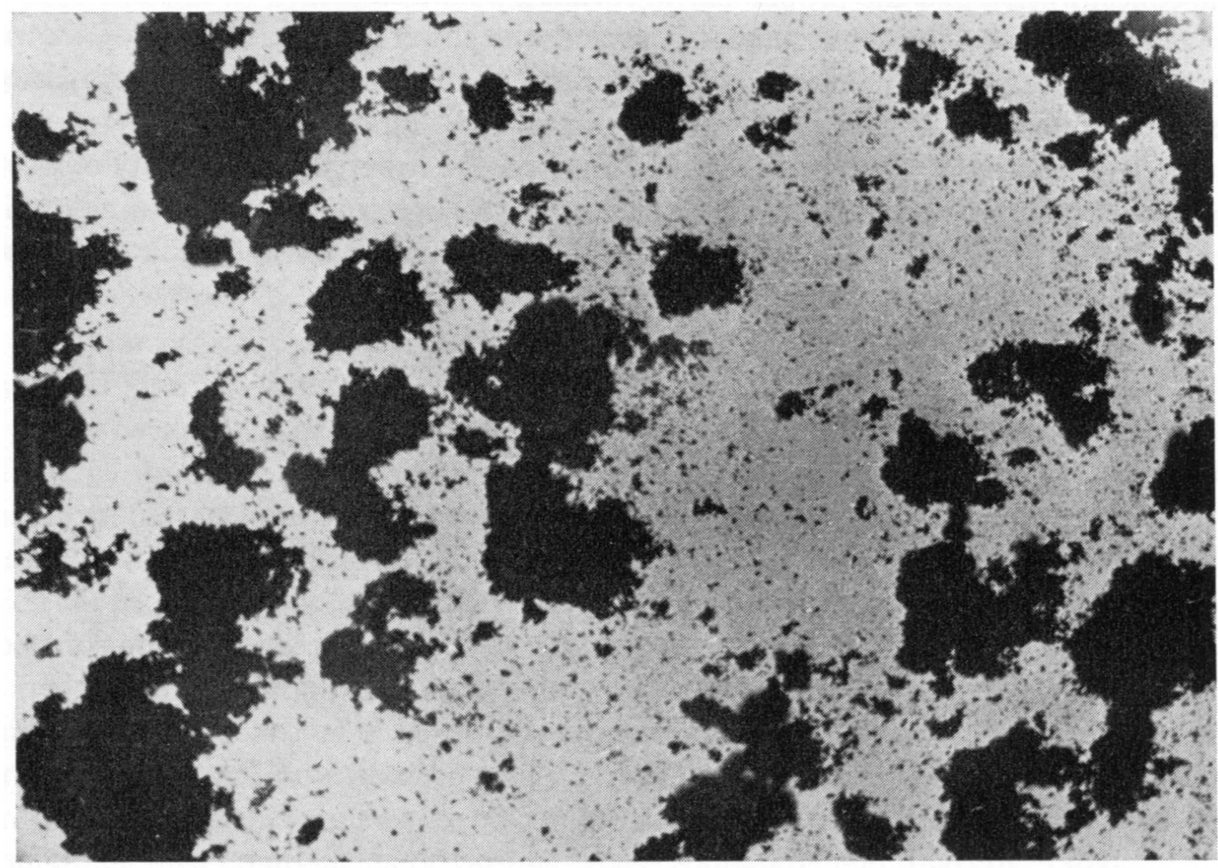

Fig. 4. Conglomerates of nickel particles. Primary particle size $2 \mu$, maximum conglomerate size 5 to $7 \mathrm{~mm}$.

following assumptions and conclusions with regard to the physical processes taking place within comets:

(1) At considerable heliocentric distances a cometary nucleus containing a dust component will have a fairly low temperature, which will favour dust-matrix formation.

(2) As the comet approaches the Sun the temperature of a nucleus enclosed in a matrix will increase, and this may result in disintegration and shedding of the matrix. This process may take the form of an explosion, leading to a sharp increase in the amount of dust in the comet's head and an outburst in brightness.

(3) Once the matrix has been shed, the flow of volatiles from the icy surface increases. This causes the nucleus to be cooled down and reestablishes conditions for matrix formation. Thus the matrix may be formed and shed periodically as the comet approaches the Sun, the frequency of the cycles increasing with time.

(4) Most of the micro-sized dust leaves the cometary nucleus, not as separate particles, but in the form of conglomerates of varying size, shape and structure. 
(5) The outflow of separate dust particles of submicron size from the ice surface is only possible at small heliocentric distances. The appearance of submicron dust at large distances from the Sun is presumably associated with some other mechanism, such as that suggested by Dolginov (1967).

(6) When conglomerate leaving the nucleus contains a volatile component, sublimation of this component may cause disintegration of the conglomerate, as well as its acceleration due to the exhaust thrust thus generated.

(7) Periodic meteor streams are remnants of comets. According to our views, meteors must be composed of conglomerates of particles. When such conglomerates enter the upper atmosphere they will be relatively bright objects, but due to their porous structure their reduced densities will be quite low.

[After the presentation of this paper a short film was shown, demonstrating some of the effects caused by sublimation of pure water ice and ice contaminated by dust particles.]

\section{References}

Beller, W.: 1963, Missiles and Rockets 12, No. 8, 22.

Boggess, A.: 1965, Space Science, London and Glasgow, p. 729.

Danielsson, L. R. and Kasai, G. H.: 1968, J. Geophys. Res. 73, 259.

Delsemme, A. H. and Swings, P.: 1952, Ann. Astrophys. 15, 1.

Dolginov, A. Z.: 1967, Astron. Zh. 44, 434.

Donn, B.: 1964, Astron. J. 69, 137.

Dorsey, N. E.: 1940, Properties of Ordinary Water-Substance, Reinhold, New York.

Föppl, H.: 1965, Weltraumfahrt 16, 139.

Haser, L. and Lüst, R.: 1966, Raumfahrtforschung 10, 196.

Kajmakov, E. A. and Sharkov, V. I.: 1969, Trudy IV Zimnej Shkoly po Kosmofizike, Apatity, part $2,15$.

Kajmakov, E. A., Sharkov, V. I., and Zhuravlev, S. S.: 1972, this Symposium, p. 316.

Potter, A. F. and del Duca, B.: 1964, Icarus 3, 103.

Roberts, J. G.: 1963, Spaceflight 5, 213.

Rosen, B. and Bredohl, H.: 1965, Techn. Humn. 62, No. 2, 5.

Rylov, V. S.: 1967, Komety $i$ Meteory No. 15, 3.

Shklovskij, I. S. and Kurt, V. G.: 1959, Iskusstv. Sputniki Zemli No. 3, 66.

Swings, P.: 1962, Space Age Astronomy, Academic Press, New York and London, p. 370.

\section{Discussion}

J. M. Witkowski: What is the density of the matrix with respect to the density of the dust?

E. A. Kajmakov: Matrix density depends on dust concentration. Actually, if the concentration exceeds $1 \%$, we can only guess that there has been ice sublimation. The dust structure in the matrix tends to reproduce the structure of the dust-laden ice. The density of the matrix may be one order of magnitude lower than that of dust-particle substance.

F. L. Whipple: I must congratulate you on your most successful and striking experiments. I deeply appreciate seeing in your film the effects I talked about 20 yr ago. 